\title{
Estimation of annual average daily traffic with optimal adjustment factors
}

Borja Alonso PhD

Assistant Professor, University of Cantabria. Department of Transport, ETS Ing de Caminos, Santander, Spain

José Luis Moura PhD

Professor of Transport, University of Cantabria. Department of Transport, ETS Ing de Caminos, Santander, Spain
Angel Ibeas PhD

Professor of Transport, University of Cantabria. Department of Transport, ETS Ing de Caminos, Santander, Spain

Juan Pablo Romero PhD

Researcher, University of Cantabria. Department of Transport, ETS Ing de Caminos, Santander, Spain

This study aimed to estimate the annual average daily traffic in inter-urban networks determining the best correlation (affinity) between the short period traffic counts and permanent traffic counters. A bi-level optimisation problem is proposed in which an agent in an upper level prefixes the affinities between short period traffic counts and permanent traffic counters stations and looks to minimise the annual average daily traffic calculation error while, in a lower level, an origin-destination (O-D) trip matrix estimation problem from traffic counts is solved. The proposed model is tested over the well-known Sioux-Falls network and applied to a real case of Cantabria (Spain) regional road network. The importance of determining appropriate affinity and the effect of localisation of permanent traffic counters stations are discussed.

\author{
Notation \\ A set of all links with traffic count \\ a link with traffic count \\ $l \quad$ network link or road section \\ $P_{i j}^{a} \quad$ fraction of trips from $i$ to $j$ using link $a$ \\ sa average daily traffic for Saturdays \\ su average daily traffic for Sundays \\ $T_{\text {annual }} \quad$ mean average daily traffic for the working days of \\ the different months \\ $T_{k} \quad$ mean average daily traffic for working days in one \\ determined month $k$ \\ $T_{16 \mathrm{~h}} \quad 16 \mathrm{~h}$ traffic \\ $T_{24 \mathrm{~h}} \quad 24 \mathrm{~h}$ traffic \\ $T=\left[T_{i j}\right] \quad$ the origin-destination matrix to be estimated \\ $\widehat{T}=\left[\widehat{T}_{i j}\right] \quad$ the vector containing the a priori trip matrix \\ $\widehat{V}_{l} \quad$ measured volume (AADT) on link $l$ \\ $V_{l}^{0} \quad$ estimated volume (AADT) on link $l$ \\ wo $\quad$ AADT for working days. \\ $\delta_{n, m} \quad$ element of the SPTC-PTC incidence matrix, taking \\ a value of 1 if SPTC $m$ is related to PTC $n$ and 0 in \\ other cases \\ $\gamma_{1}, \gamma_{2} \quad$ weighting scalars
}

\section{Introduction}

Annual average daily traffic (AADT) is a key parameter of road design. However, its estimation is a difficult problem because the only way to obtain its real value is by measuring 365 days per year and $24 \mathrm{~h}$ per day.

Obviously, this methodology would be unviable for any govern- ment. For this reason only a few stations measure all the year and short period measurements are used to complete this information. Thus, the network of traffic counting stations is classified into two different types.

- Permanent traffic count (PTC). Observation is made all year round by electronic counters using magnetic induction loops fitted with time registers and able to distinguish between heavy and light vehicles.

- Short period traffic counts (SPTC). Complementary traffic counting at certain points of the road network where measurements are taken for $16 \mathrm{~h}$ (from 0600 to $2200 \mathrm{~h}$ ) for one working day only.

Given that the objective is to obtain AADT values from the SPTC values, an expansion of their measurements must be made $(F)$. In Spain, this is done by extracting three types of expansion factors associated with each PTC. Each of these three factors is calculated in the following manner.

- Nocturnality factor $N$ : The relationship between $24 \mathrm{~h}$ traffic $\left(T_{24} \mathrm{~h}\right)$ and $16 \mathrm{~h}$ traffic $\left(T_{16 \mathrm{~h}}\right)$.

1. $\quad N=T_{24 \mathrm{~h}} / T_{16 \mathrm{~h}}$

Seasonal factor $L$ : Transforms the average daily traffic for any one working day into the AADT for working days. It is obtained by dividing the average daily traffic for working 
days by the average daily traffic for working days in one determined month $k$.

$$
\text { 2. } \quad L_{k}=T_{\text {annual }} / T_{k}
$$

Weekend factor $S$ : This transforms the average daily traffic for working days into the AADT, introducing the effect of Saturdays and Sundays. It is calculated using the following expression:

$$
\text { 3. } S=(5+(s a / w o)+(s u / w o)) / 7
$$

- Final factor $F$ : This transforms the traffic count done at a SPTC over $16 \mathrm{~h}$ into the AADT.

$$
\text { 4. } F=N \cdot L \cdot S
$$

Each SPTC is associated to a unique PTC (this is called 'affinity') and multiplying the traffic count at the SPTC by the factor $(F)$ at point PTC produces the AADT for the SPTC.

Thus the problem is how to find the best affinity between SPTC and PTC and, furthermore, how many SPTC are really needed for a correct estimate of the AADT across the entire network. For example, the authorities in Spain who are responsible for deciding this affinity, normally base the decision purely on empirical experience and on data they have inherited from historical configurations that are very often obsolete.

The international literature was consulted to try and sort this out. The work done by Lingras and Adamo (1996) showed the results of a statistical comparison between the traditional method and a genetic algorithm to classify sections of motorway by analysing a temporal traffic pattern. This improvement allows for a better analysis of traffic by providing a more accurate calculation of the AADT.

Sharma et al. (1996) studied the statistical precision of obtaining AADT from SPTC stations. A high number of PTC locations were studied on the Minnesota motorway network to look at the effects of different factors on the error in the calculation, narrowly related with the affinity which establishes the parameters for grouping the PTCs. It was concluded that the results are highly sensitive to the chosen definition of affinity.

Lam and Xu (2000) adopted the neural network method for the estimation of AADT from SPTC stations and they compared their estimation with the one obtained by regression. Later Sharma et al. (2001) used neural networks to calculate AADT on lowvolume roads from SPTCs. They also describe some advantages associated with calculating from neuronal networks rather than the more traditional method using factors.
Yang and Davis (2002) used Bayesian techniques for calculating classified mean daily traffic and also analysed the effect of how long a short duration traffic count took. McCord et al. (2003) proposed an alternative methodology in which AADT was estimated from a single image of a road segment and, based on this model, Jiang et al. (2006) proposed a different approach that exploited existing imagery of highway segments and earlier year coverage counts.

The model proposed herein presents a different approach because it is based on an origin-destination (O-D) matrix estimation from PTC and SPTC data which reproduces the AADT on the network. Thus the model determines the optimal affinity between SPTC and PTC and, therefore, the number and allocation of these SPTC for each PTC, minimising the error over validation measurements. Until now, only the work of Wang (2012), who used parcel-level travel demand modelling, had used travel demand modelling for this estimation.

The next section presents the proposed model. A test application is presented for the well-known Sioux-Falls network along with an analysis of results and discussion and this is followed by a real study case for the road network of Cantabria (Spain). Finally, the most important conclusions from this work are presented.

\section{Proposed model}

A mathematical bi-level optimisation model is proposed for solving the problem of determining the optimal affinity between SPTC and PTC. At the model's upper level, an agent varies these affinities and the lower level solves an $\mathrm{O}-\mathrm{D}$ matrix estimation problem from traffic counts.

Suppose a road network has $N$ PTC stations and $M$ SPTC stations. The adjustment factor (affinity) between PTC and SPTC stations is unique; in other words, each SPTC station has only one comparable PTC station. Hence an incidence matrix or affinity matrix $[N \times M]$ can be created, the elements of which, $\delta_{n, m}$, are equal to 1 if the SPTC $m$ is related to the PTC $n$, and 0 otherwise. Each of the annual counts from every PTC station is assigned an AADT and $F$ value. Similarly, at each SPTC station, where traffic was counted for $16 \mathrm{~h}$, by applying the $F$ factor of the related PTC station the final AADT value of all the SPTC stations was derived.

Then an O-D trip matrix is estimated from the AADT values. In traffic models one well-known problem is the trip matrix estimation, where some observations (link flows) are taken and the O-D flows are estimated (Ashok and Ben-Akiva, 2000; Cascetta, 1984; Cascetta and Nguyen, 1988; Doblas and Benitez, 2005; Hazelton, 2000; Lo et al., 1996; Maher and Zhang, 1999 or Yang et al., 1992).

The estimated matrix provides network flows, which can then be compared with real observable counts. Therefore, the problem is to find the best affinity matrix (with the constraint that an SPTC station can only be referred to one PTC station) to calculate the O-D trip matrix with minimum error. The mean absolute error (MAE) between the estimated and observed flows was used. 
Upper level

5. $\quad \min \mathrm{MAE} \%=\frac{\sum_{a \in A}\left|\widehat{V}_{a}-V_{a}^{0}\right|}{\sum_{a \in A} \widehat{V}_{a}} \cdot 100$

such that

6. $\sum_{n \in N} \delta_{n, m}=1, \forall m \in M$

7. $\delta_{n, m} \in\{0,1\}$

Lower level

$$
\begin{aligned}
\min Z(t, v)= & \gamma_{1} \sum_{i j} T_{i j}\left(\ln \frac{T_{i j}}{\widehat{T}_{i j}}-1+\ln \widehat{T}\right) \\
& +\gamma_{2} \sum_{a \in A} V_{a}\left(\ln \frac{V_{a}}{\widehat{V}_{a}}-1\right)
\end{aligned}
$$

9. $\widehat{V}_{a}=\sum_{i j} T_{i j} \cdot P_{i j}^{a}, \quad a \in A$

Constraint 6 ensures that each SPTC is only associated with one PTC. Equations 8 and 9 correspond to the well-known method of O-D matrix estimation from traffic counts based on maximising entropy.

The methodology is shown in Figure 1 and can be summarised in the following steps.

1. The starting point is a series of data: Initial O-D trip matrix, group of PTC and SPTC stations and an initial matrix of adjustment factors (affinities).

2. The initial matrix of adjustment factors is used to calculate the AADT for the PTC and SPTC stations.

3. O-D matrix estimation from traffic counts

4. The modelled flows are then compared with the validation flows and the MAE is calculated. If the MAE value drops with respect to the previous iteration value then another matrix of adjustment factors is looked for and the calculation returns to step 2. If the error does not drop then the latest $\mathrm{O}-\mathrm{D}$ trip matrix and its associated matrix of adjustment factors are optimal. A generic genetic algorithm that realises

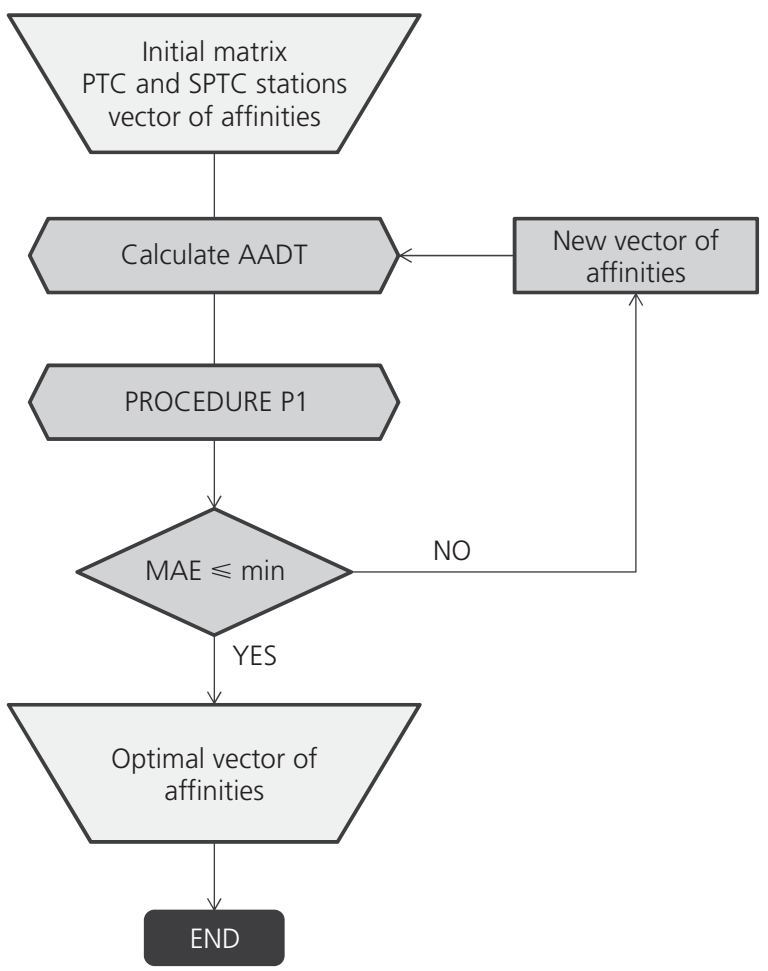

PROCEDURE P1

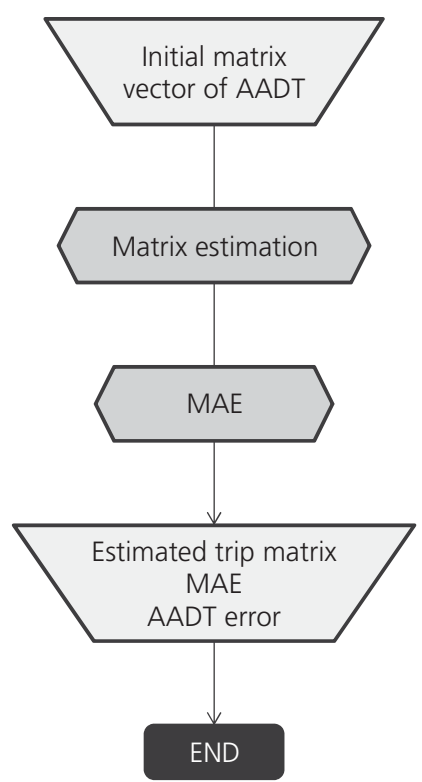

Figure 1. Methodology 
Transport

Volume 168 Issue TR5
Estimation of annual average daily traffic

with optimal adjustment factors

Alonso, Moura, Ibeas and Romero a heuristic search, which departs from an initial solution of the matrix of adjustment factors and generates a new solution exchanging the initial solution (Mathe and Grefenstette,

2004), has been used in this point.

\section{Sioux-Falls network test application}

The methodology was applied to the well-known Sioux Falls network, composed of 24 zones, 24 nodes and 76 links. The necessary data are: the initial trip matrix and the group of PTC stations with their $F$ values as well as the group of SPTC stations. Furthermore, it was assumed the target ('real') trip matrix is known. These data are shown in Table 1 along with the first configuration of affinities (an initial affinity based on the criteria of closeness).

After applying the model, the affinity matrix which minimises the error in the flow on links is shown in the same Table 1, and its network representation is shown in Figure 2. In addition to managing to minimise the error it is interesting to also study the error committed in the estimations of AADT for the SPTC stations. Figure 3 shows how the estimation errors evolve for the $\mathrm{O}-\mathrm{D}$ matrix and AADT from the affinity with greatest error to the optimal one. Both errors are seen to descend but the descent is more profound for AADT (from $18 \cdot 17$ to $7 \cdot 16 \%$ ) than for the O-D matrix (from 18.09 to $14 \cdot 3 \%$ ).

Once the optimal affinities between SPTC and PTC have been obtained, it may be interesting to analyse how the different affinities criteria affect the results in comparison with the optimal solution. The AADT errors were compared for $(a)$ the initial affinity case (closeness), (b) the case of all the SPTC having affinity to PTC1, (c) the case of all the SPTC having affinity to PTC2 and $(d)$ the optimal case which minimises MAE. Figure 4 shows the AADT relative error of each SPTC, and it can be seen that the optimal case is clearly better than the others. Moreover, significant differences in the AADT error obtained are seen if the affinities are not set correctly, which certainly contributes to a biased estimate with serious consequences during the planning phase.

From these results a second-best optimal traffic counts algorithm with budgetary constraint (Ehlert et al., 2006) or a heuristic iterative method such as the stepwise selection method can be applied to reduce the number of SPTC. According to the previous example, the same subset of possible SPTC links was used and the maximum number of SPTC to be included was fixed to only six stations (equal installing costs are assumed). The final location of SPTC and their affinities are also shown in Figure 2 and can be compared with the results of the previous cases. The MAE obtained increased slightly up to $9 \cdot 11 \%$ for AADT (from $7 \cdot 16 \%$ ) and $15 \cdot 18 \%$ for the O-D matrix (from $14 \cdot 3 \%$ ); however, these values are still accurate considering the number of SPTC was $40 \%$ lower.

\section{Discussion}

Looking at the results of the model for AADT estimation and trip matrix estimation in inter-urban networks (determining the best adjustment factor between PTC and SPTC), the MAE and the error for AADT estimation can change, due to the following factors.

- The well-known problem of irrelevant and inconsistent information: the inclusion of a new SPTC into the optimal set may mean that a previously chosen SPTC is providing irrelevant and/or inconsistent information. The flow represented by SPTC_ $\{i\}$ is represented in an equal or better

\begin{tabular}{|c|c|c|c|c|c|c|c|c|}
\hline & & \multicolumn{3}{|c|}{ Permanent traffic counter } & \multirow[b]{2}{*}{ AADT } & & \multirow[b]{2}{*}{ PTC 1} & \multirow[b]{2}{*}{ PTC2 } \\
\hline & & EP1_1 & EP1_2 & Traffic count $16 \mathrm{~h}$ & & & & \\
\hline \multirow{12}{*}{$\begin{array}{l}\text { Short } \\
\text { traffic } \\
\text { period } \\
\text { counts }\end{array}$} & SPTC1 & 1 & 0 & 18850 & 28087 & SPTC1 & 0 & 1 \\
\hline & SPTC2 & 1 & 0 & 18842 & 28075 & SPTC2 & 1 & 0 \\
\hline & SPTC3 & 1 & 0 & 16232 & 19077 & SPTC3 & 0 & 1 \\
\hline & SPTC4 & 1 & 0 & 16518 & 24612 & SPTC4 & 1 & 0 \\
\hline & SPTC5 & 1 & 0 & 30672 & 45701 & SPTC5 & 1 & 0 \\
\hline & SPTC6 & 0 & 1 & 26224 & 30813 & SPTC6 & 0 & 1 \\
\hline & SPTC7 & 0 & 1 & 11788 & 13851 & SPTC7 & 1 & 0 \\
\hline & SPTC8 & 0 & 1 & 24420 & 36386 & SPTC8 & 1 & 0 \\
\hline & SPTC9 & 0 & 1 & 11626 & 17323 & SPTC9 & 0 & 1 \\
\hline & SPTC10 & 0 & 1 & 6486 & 7621 & SPTC10 & 0 & 1 \\
\hline & AADT & 22500 & 20400 & & & & & \\
\hline & $\mathrm{F}$ & 1.49 & $1 \cdot 17$ & & & & & \\
\hline
\end{tabular}

Table 1. Initial and optimal matrix of affinity between PTC and SPTS, and AADT values for Sioux Falls network 


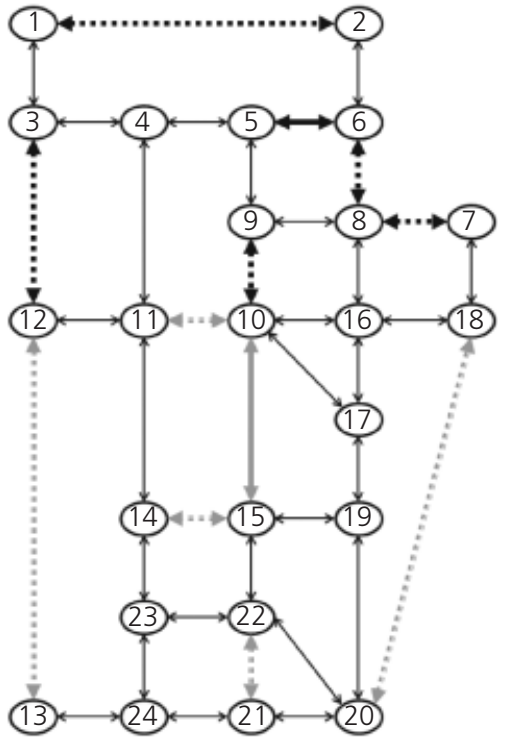

(a)

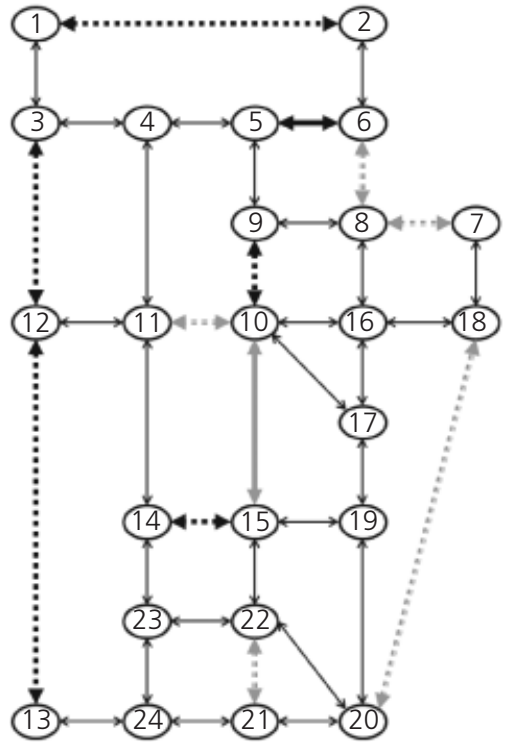

(b)

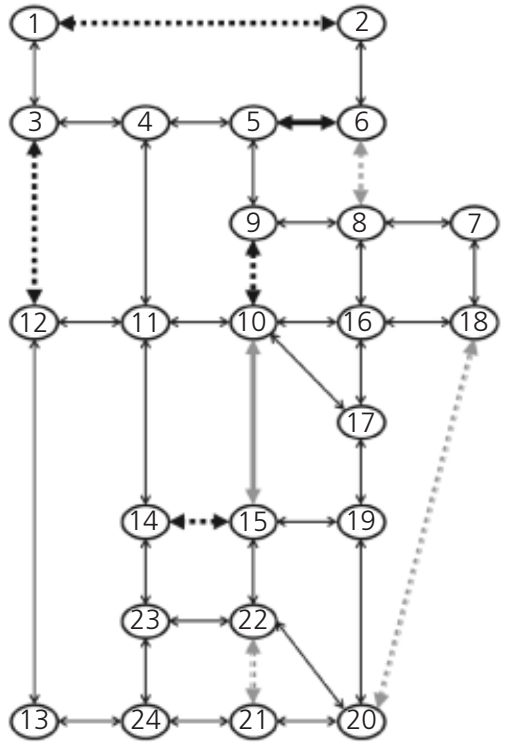

(c)
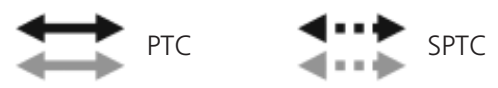

Figure 2. PTC stations with SPTC stations related: (a) initial case;

(b) optimal situation; (c) minimum SPTC required

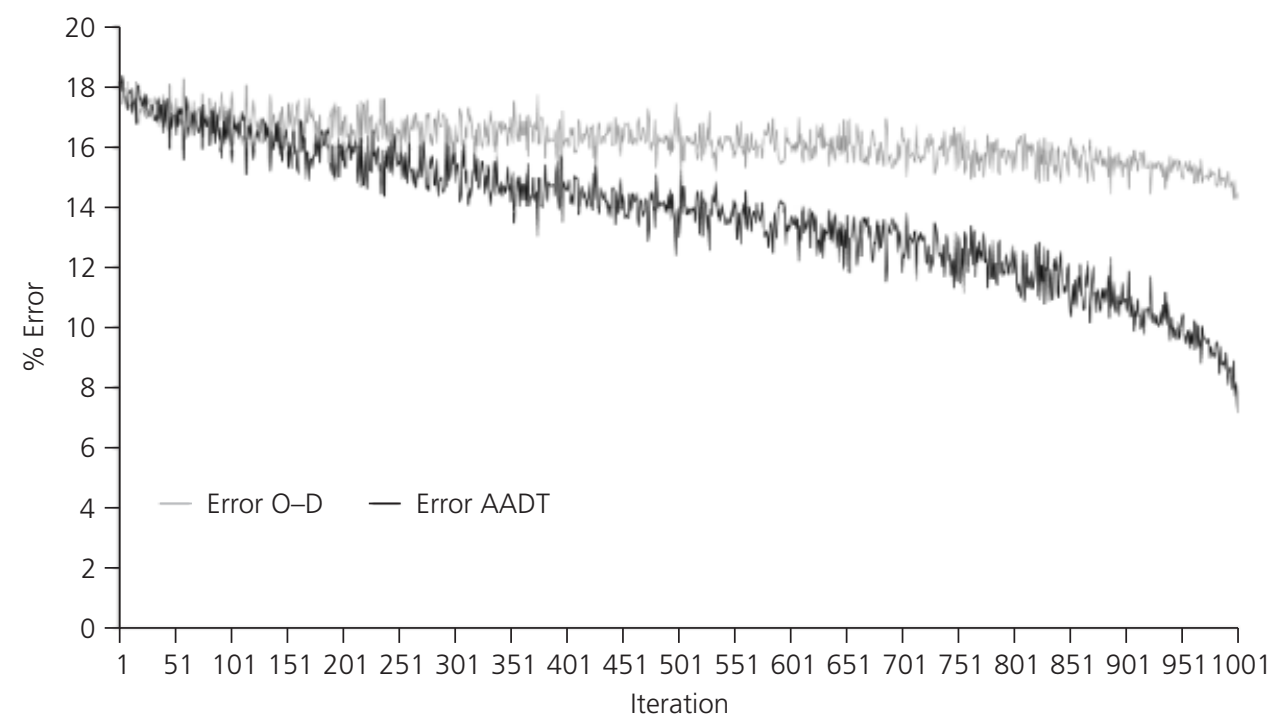

Figure 3. Estimation errors for the O-D matrix and AADT

way by one or more of the SPTCs in the group (including the recently selected one), but they provide additional information which SPTC_ $i$ does not provide.

- Specifically in the case of inter-urban networks, the error associated with the AADT values for the SPTC stations is related to estimations obtained from the definition of adjustment factor, unlike for the urban networks where typically all the groups of traffic counts are from observed data (there could only be errors associated with observation or seasonal inconsistency, but never about estimation). This demonstrates the importance of correctly defining the adjustment factor between the PTC and SPTC stations.

Another question worth mentioning is the effect of the predetermined location of the PTC stations and how this can affect 


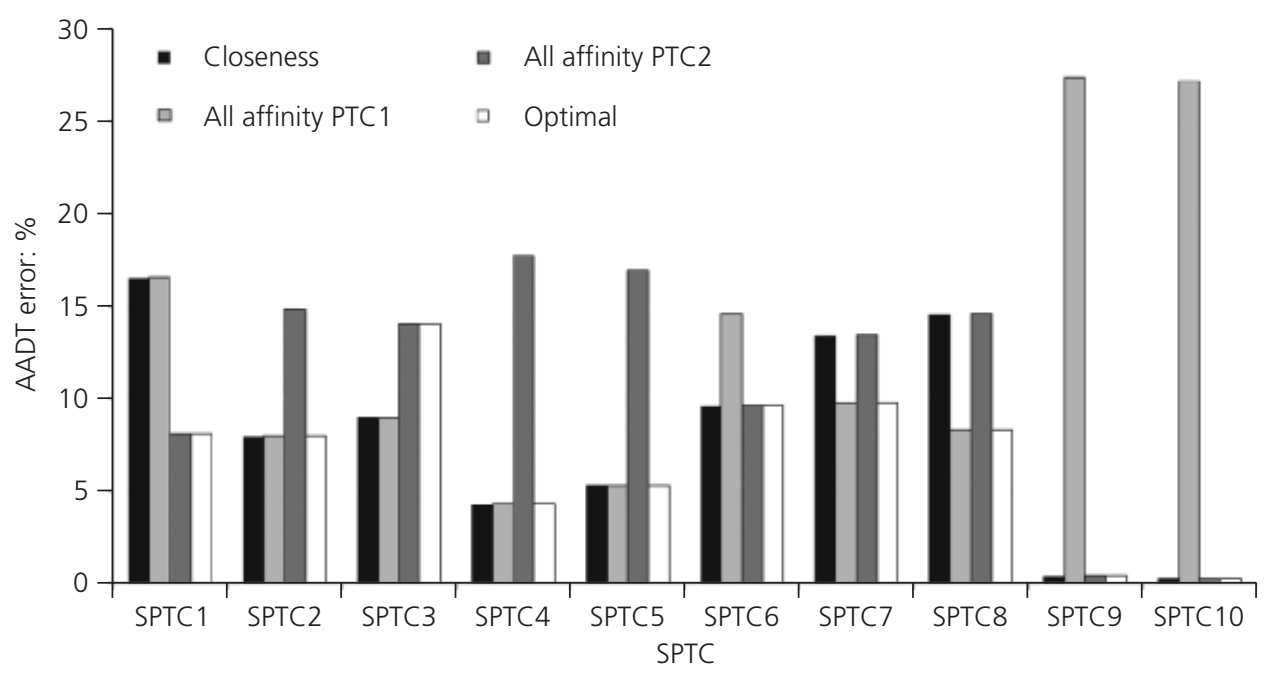

Figure 4. Accumulated error in AADT

the optimal location of SPTC and the affinity matrix. In the cases mentioned above they were situated at two locations of highvolume traffic without using any quantitative criteria.

Several approaches to solve the optimal location of traffic counts problem follow the selection rules defined by Yang and Zhou (1998).

- OD covering rule: a certain proportion of trips between each OD pair should be observable.

- Maximal flow fraction rule: for a particular OD pair, links with the highest fraction of that OD flow should be selected.

- Maximal flow interception rule: the set of links which intercept the maximum number of OD movements should be selected.

- Link independence rule: links with linearly independent flows should be selected.

At this point it is worth asking if it would be more beneficial to locate the PTC at places with the highest volumes of traffic or which have the highest sum of $P_{i j}^{a}$ or what happens if the PTC is located by applying an optimal location of traffic counts algorithm (OLTC), and which of these shows the lowest MAE, with a maximum of 10 SPTC to be located (equal installing costs are assumed again). The three cases were analysed and the final location and affinities are represented in Figure 5. Both the lowest error in the AADT values and the MAE of the target $\mathrm{O}-\mathrm{D}$ matrix appear in the case of locating the PTC station following the OLTC results (Table 2).

As can be seen, there are three key problems during the planning process of traffic data collection, each of them depends on the others.

- P1. Optimal location of PTC: this problem is beyond the scope of this research. Its practical application is not easy and is subject to many conditions because many authorities have already located their traffic counters. However, this problem can be modelled as an optimal location of traffic counts problem subject to budgetary constraint (Chung, 2001).

- P2. Optimal location and number of SPTC: as stated in the previous section, this problem can be considered as a secondbest count location problem, taking into account the existing PTC and subject to budgetary constraint (Ehlert et al., 2006).

- P3. To find the best affinity between SPTC and PTC.

This study has focused on P3, which depends on P2 and P1. Furthermore, the output of P3 can be used for solving P2. An example of this application has been shown in Section 3. In turn, P2 depends on P1, therefore, P1 affects to the others because the PTC are taken into account during all planning phases.

This section has proved that deterministic criteria for locating PTC, even with the optimal affinities of the SPTC, affect the results, both in AADT and the $\mathrm{O}-\mathrm{D}$ matrix estimation. Thus, it was found that PTC location based on optimisation and location models was more efficient and achieved better results in successive models to solve $\mathrm{P} 3$ and $\mathrm{P} 2$, respectively.

\section{Application to a real case: regional road network of Cantabria (Spain)}

Finally, the methodology was applied to a real case: the regional road network of Cantabria (Spain), composed of 273 zones, 1367 nodes and 3210 links. The current counts stations are identified in Figure 6: 16 PTC and 592 SPTC (both directions: 1184 links), distributed as they appear in the figure and with adjustment factors defined empirically, without any optimisation process. At the same time 41 ATC were deployed for measuring AADT and to validate the model.

As soon as the model was applied, the MAE descent could be seen across the iterations of the algorithm. The algorithm 


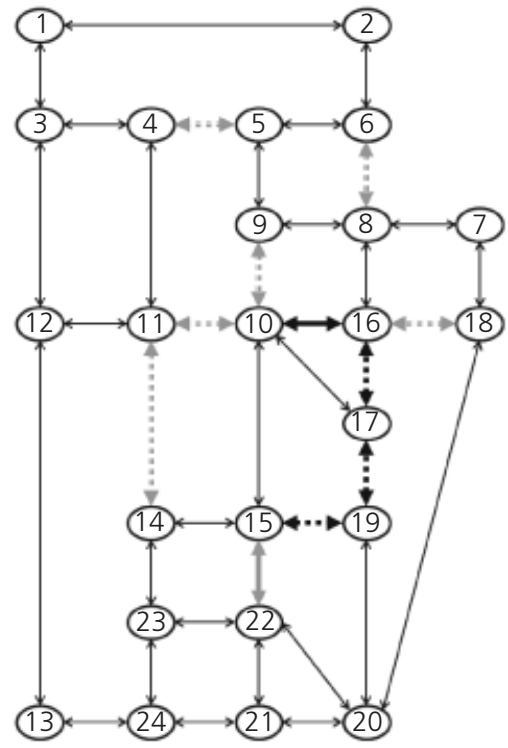

(a)

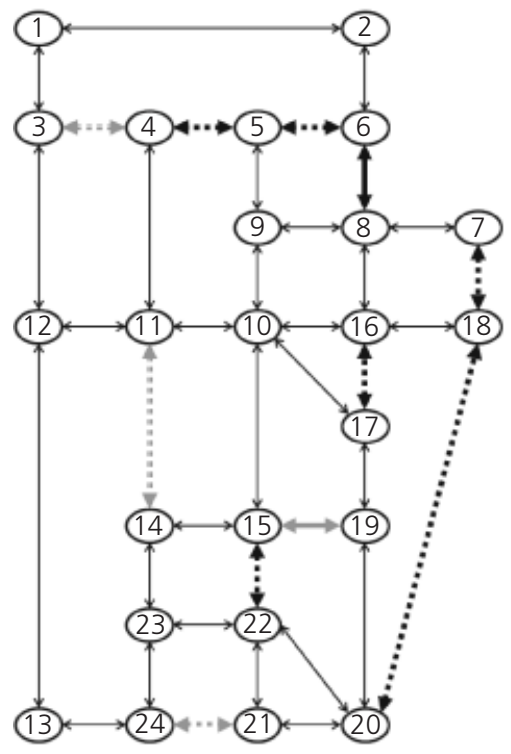

(b)

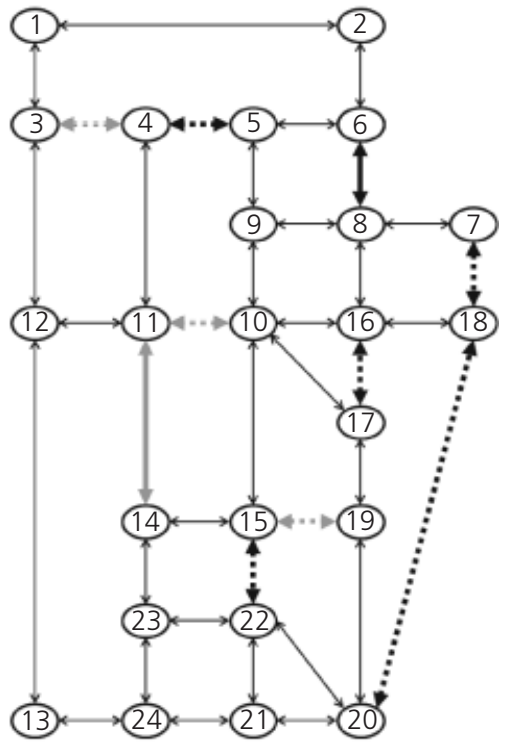

(c)
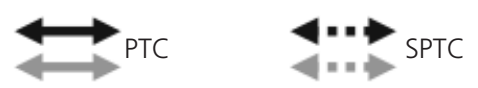

Figure 5. PTC stations with SPTC stations related: (a) in the case for locating PTCs in links with highest traffic volume; (b) in the case for locating PTCs in links with highest sum $P_{i j}^{a}$; (c) in the case to be located solving an OLTC problem

\begin{tabular}{lcc}
\hline & MAE & Average error AADT \\
\hline Initial case & 14.27 & $7 \cdot 16$ \\
Highest traffic volume & 14.00 & 5.47 \\
Highest sum of $P_{i j}$ & 13.86 & $5 \cdot 11$ \\
OLTC & 12.27 & 4.41
\end{tabular}

Table 2. MAE and average error AADT in all cases

began with the initial adjustment factors (current) and finally a new redistribution of adjustment factors was reached. The MAE diminished by $4 \cdot 3 \%$ (from $20 \cdot 9$ to $16 \cdot 5 \%$ ). Figure 7 shows the evolution of the algorithm up to reaching the optimal solution.

By considering the AADT estimation and comparing both situations (initial situation with current matrix of adjustment factors and final situation with proposed new adjustment factors), all the SPTC (592) were checked and this analysis detected that the biggest difference between the AADT values estimated was $48 \%$ (absolute value).

Therefore, the model optimised the related SPTC to the PTC in both number and situation. In this analysis, the model found other relations between PTC and SPTC in which there were related types of roads with the same characteristics or there were roads of the same category, in contrast to the initial situation in which this relation was based on closeness criteria.

Finally, as stated in previous sections, from the results given by this model in terms of affinities, it is feasible to apply a model for determination of the optimal location and number of traffic counts (SPTC). Its practical application using the stepwise selection method over the same network and its benefits can be found in Alonso et al. (2013).

\section{Conclusion}

The proposed model determines the best adjustment factor (affinity) between PTC and SPTC based on an O-D matrix estimation from PTC and SPTC data which reproduces the AADT on the network.

The application test over the Sioux-Falls network has shown the difference in values of AADT estimation based on different affinities between SPTC and PTC, reaching double the mean absolute error.

Furthermore, it has proved that the location of PTC influences the final results and the minimum error achieved. The best results were obtained from the application of an optimisation model for locating traffic counts, maximising $\mathrm{O}-\mathrm{D}$ coverage. This is an 


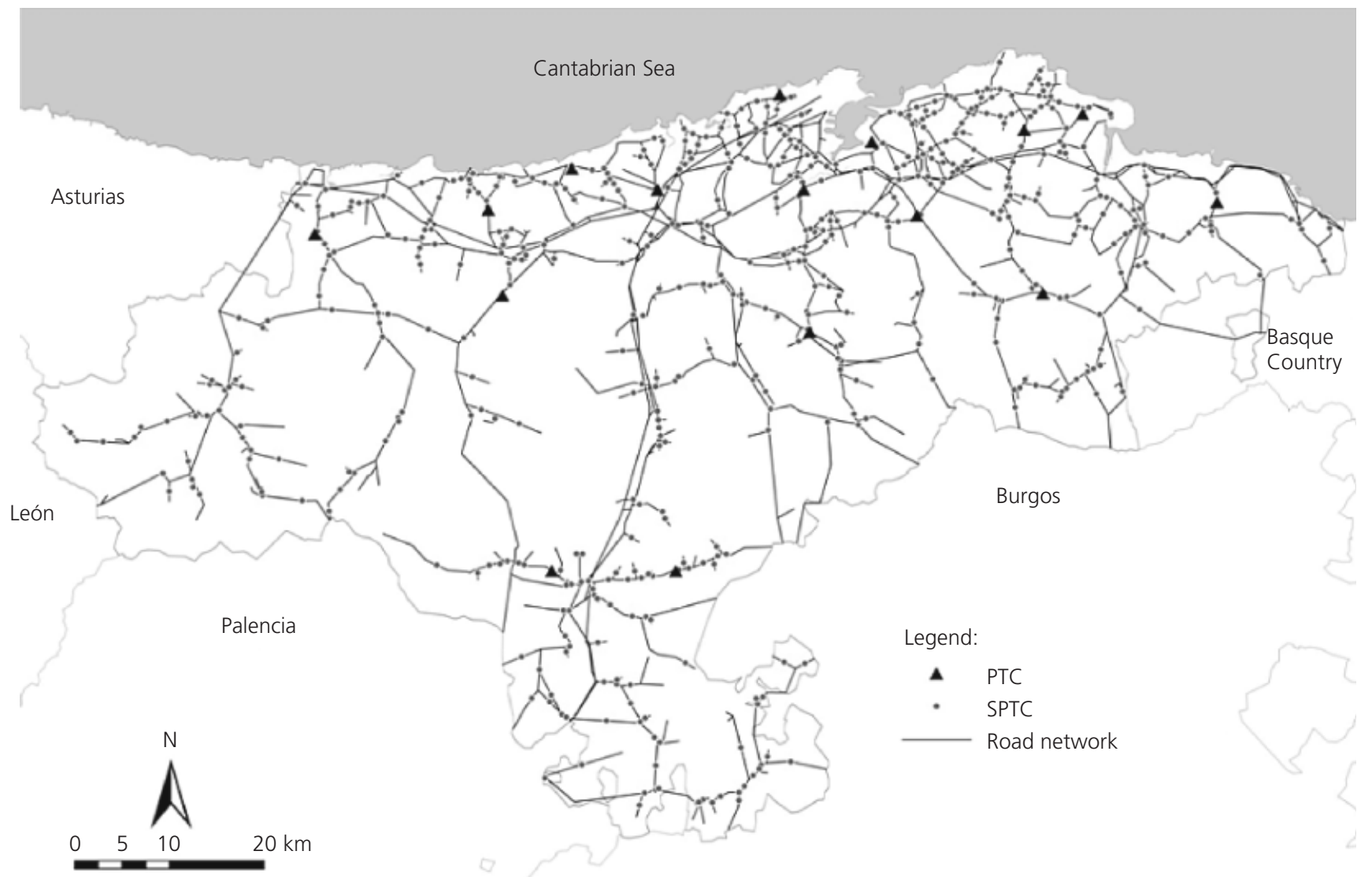

Figure 6. Current counts stations in regional road network of Cantabria

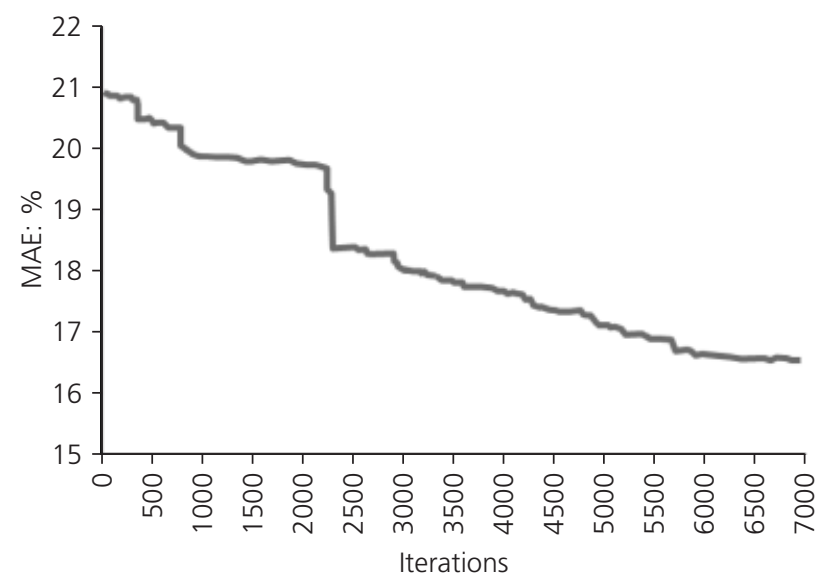

Figure 7. Evolution of the algorithm up to reaching the optimal solution

important issue because, as has been seen, this location strongly affects the final affinities of the SPTC and their locations.

Once the methodology had been tested in a sample network, it was applied to a real case: the regional road network of Cantabria (Spain). The mean absolute error (MAE) diminished by $4.3 \%$ (from $17 \cdot 7$ to $13.4 \%$ ), certifying that the new adjustment factors proposal better reproduces the OD trips.

Finally, the initial situation with current matrix of adjustment factors and the final situation with new adjustment factors were plotted, analysing the produced changes. In this analysis, the model found other relations between PTC and SPTC in which there are related types of roads with the same characteristics or there are roads of the same category, in contrast to the initial situation in which this relation was based on closeness criteria.

\section{REFERENCES}

Alonso B, Moura JL, Ibeas A and Dell'Olio L (2013) Using O-D matrices for road network management decision making. Transport 28(1): 31-37.

Ashok K and Ben-Akiva M (2000) Alternative approaches for real-time estimation and prediction of time-dependent origindestination flows. Transportation Science 34(1): 21-36.

Cascetta E (1984) Estimation of trip matrices from traffic counts 
Estimation of annual average daily traffic

with optimal adjustment factors

Alonso, Moura, Ibeas and Romero and survey data: a generalized least squares estimator. Transportation Research Part B 18(4): 289-299.

Cascetta E and Nguyen S (1988) A unified framework for estimating or updating origin/destination matrices from traffic counts. Transportation Research Part B 22(6): 437-455.

Chung I-H (2001) An Optimum Sampling Framework for Estimating Trip Matrices from Day-to-day Traffic Counts. $\mathrm{PhD}$ thesis, University of Leeds, Leeds, UK.

Doblas J and Benitez FG (2005) An approach to estimating and updating origin-destination matrices based upon traffic counts preserving the prior structure of a survey matrix. Transportation Research Part B 39(7): 565-591.

Ehlert A, Bell MGH and Grosso S (2006) The optimization of traffic count locations in road networks. Transportation Research Part B 40(6): 460-479.

Hazelton ML (2000) Estimation of origin-destination matrices from link flows on congested networks. Transportation Research Part B 34(7): 549-566.

Jiang Z, McCord MR and Goel PK (2006) Improved AADT estimation by combining information in image-and groundbased traffic data. Journal of Transportation Engineering ASCE 132(7): 523-600.

Lam WHK and XU J (2000) Estimation of AADT from short period counts in Hong Kong - a comparison between neural network method and regression analysis. Journal of Advanced Transportation 34(2): 249-268.

Lingras P and Adamo M (1996) Average and peak traffic volume: neural nets, regression, factor approaches. Journal of Computing in Civil Engineering 10(4): 300-306.

Lo HP, Zhang N and Lam WHK (1996) Estimation of an origindestination matrix with random link choice proportions: a statistical approach. Transportation Research Part B 30(4): 309-324.
Maher MJ and Zhang X (1999) Algorithms for the solution of the congested trip matrix estimation problem. In Transportation and Traffic Theory, Proceedings of the 14th International Symposium on Transportation and Traffic Theory. Elsevier, Amsterdam, the Netherlands, pp. 445-469.

Mathe E and Grefenstette J (2004) Polyoptimizing genetic algorithms for feature subset selection. Interface: Classification and Clustering, Proceedings of the 36th Symposium on the Interface, Baltimore, MD, USA.

McCord M, Yang Y, Jiang Z, Coifman B and Goel P (2003) Estimating AADT from satellite imagery and air photographs: empirical results. Transportation Research Record 1855: 136-142.

Sharma S, Gulati B and Rizak S (1996) Statewide traffic volume studies and precision of AADT estimates. Journal of Transportation Engineering ASCE 122(6): 430-439.

Sharma S, Lingras P, Xu F and Kilburn P (2001) Application of neural networks to estimate AADT on low-volume roads. Journal of Transportation Engineering ASCE 127(5): 426-432.

Wang T (2012) Improved annual average daily traffic (AADT) estimation for local roads using parcel-level travel demand modeling. FIU Electronic Theses and Dissertations. Florida International University, Florida, USA, Paper 623.

Yang S and Davis GA (2002) Bayesian estimation of classified mean daily traffic. Transportation Research Part A 36(4): 365-382.

Yang $\mathrm{H}$ and Zhou J (1998) Optimal traffic counting locations for origin-destination matrix estimation. Transportation Research Part B 32(2): 109-126.

Yang H, Sasaki T, lida Y and Asakura Y (1992) Estimation of origin-destination matrices from link traffic counts on congested networks. Transportation Research Part B 26(6): $417-434$.

\footnotetext{
WHAT DO YOU THINK?

To discuss this paper, please email up to 500 words to the editor at journals@ice.org.uk. Your contribution will be forwarded to the author(s) for a reply and, if considered appropriate by the editorial panel, will be published as a discussion in a future issue of the journal.

Proceedings journals rely entirely on contributions sent in by civil engineering professionals, academics and students. Papers should be 2000-5000 words long (briefing papers should be 1000-2000 words long), with adequate illustrations and references. You can submit your paper online via www.icevirtuallibrary.com/content/journals, where you will also find detailed author guidelines.
} 